\title{
EXIGENCIAS PROFESIONALES
}

\author{
DEL ANIMADOR/A: COMPETENCIAS CLAVE
}

\section{PROFESSIONAL REQUIREMENTS}

FOR A SOCIOCULTURAL ANIMATOR: KEY COMPETENCES

\section{REQUISITOS PROFISSIONAIS \\ PARA UM ANIMADOR SOCIOCULTURAL: COMPETÊNCIAS BÁSICAS}

\author{
Ma Luisa Sarrate Capdevila \\ José Luis García Llamas \\ Gloria Pérez Serrano
}

Universidad Nacional de Educación a Distancia, España

RESUMEN: Este artículo es fruto de un proyecto financiado por la $\mathrm{AECID}^{1}$ sobre Intervención Educativa en Contextos Sociales y aborda una dimensión específica de la misma.

Los profesionales de la animación sociocultural reclaman un mayor reconocimiento puesto que contribuyen a resolver múltiples problemas. Han tenido la habilidad de abrir un espacio profesional específico, a lo que ha contribuido que su formación haya alcanzado nivel universitario. Su curriculum incluye aptitudes, habilidades y destrezas para ejercer una labor vinculada a diversos escenarios y contextos.

Esta investigación persigue, por un lado, identificar las competencias clave del animador/a ${ }^{2}$ que contribuyen a la práctica eficaz de su profesión y, por otro, detectar sus necesidades formativas.

Se ha utilizado una metodología multimétodo. Se elaboró un cuestionario para animadores socioculturales de todo el estado español, con una fiabilidad del 0,930, alpha de Cronbach. Se efectuaron entrevistas semiestructuradas a informantes claves. Se recibieron 376 cuestionarios. El tratamiento estadístico se realizó con el programa SPSS para los datos cuantitativos, entre ellos el análisis de varianza y factorial. Para los cualitativos se empleó el Atlas-ti.

Para el tratamiento de los resultados las competencias se sistematizaron en tres categorías: metodológicas, de planificación y genéricas, constatándose que los animadores las desempeñan en alto grado. También se han identificado las que precisan mejorar. El análisis factorial confirmatorio refleja que la propuesta inicial de tres campos vinculados a la actividad profesional, se ha transformado en cuatro en el trabajo empírico. Existe una buena identificación con las competencias metodológicas y de planificación, mientras que las genéricas se han desglosado en dos: genéricas y transversales. Todas ellas identifican el perfil de este profesional, fuente obligada de consulta. 
PALABRAS CLAVE: Animador/a sociocultural, competencias, intervención, profesión, formación.

ABSTRACT: This article comes from a project sponsored by $A E C I D^{3}$ of Educational Intervention on Social contexts and it approaches a specific dimension.

As they contribute to solve several problems, sociocultural professionals claim for higher recognition. Given their superior studies they have been able to open a professional specific space. Their CV now includes aptitudes, abilities and skills to perform a job linked to several scenarios and contexts.

The goals of this research are either to identify the key competences of the sociocultural animator that contribute to work efficiently and to detect their educational needs.

A multi methodological approach has been used. A survey was made to sociocultural animators all over Spain, with a reliability of 0,930 Cronbach's alpha. Semi organized interviews were made to key informants. 376 surveys were received back. The SSP program was used for statistical treatment for quantitative data, including variance and factorial analysis. Atlas-ti was used for qualitative data.

In order to classify the results, competences were divided into three categories: methodological, planning and generic, confirming that the sociocultural workers have them on a high degree. The factor analysis reflects that the three initially proposed working fields linked to professional performance became four with the empirical work. There is a good identification between the methodological and planning competences, while the generic is divided into: generic and transversal. A combination of them creates the professional profile, a compulsory reference source.

KEY WORDS: Sociocultural animator, competences, intervention, profession, education.
RESUMO: Como eles contribuem para resolver vários problemas, os profissionais socioculturais reivindicar maior reconhecimento. Dado os seus estudos superiores têm sido capazes de abrir um espaço específico profissional. Seu currículo inclui agora aptidões, habilidades e competências para realizar um trabalho ligado a diversos cenários e contextos.

Os objetivos desta pesquisa são tanto para identificar as competências essenciais do animador sociocultural que contribuam para trabalhar de forma eficiente e detectar suas necessidades educacionais.

Foi utilizada uma abordagem multi metodológica. A pesquisa foi feita para animadores socioculturais em toda a Espanha, com uma confiabilidade de alfa de Cronbach 0930. Entrevistas semi-organizadas foram feitos com informantes-chave. 376 inquéritos foram recebidos de volta. O programa SSP foi utilizado para o tratamento estatístico dos dados quantitativos, incluindo variância e análise fatorial. Atlas-ti foi usado para dados qualitativos.

Para classificar os resultados, as competências foram divididas em três categorias: metodologia, planejamento e genérico, confirmando que os trabaIhadores socioculturais tê-los em um alto grau. A análise fatorial reflete que os três campos de trabaIho propostos inicialmente ligados ao desempenho profissional tornou-se quatro com o trabalho empírico. Há uma boa identificação entre as competências metodológicas e de planejamento, enquanto o genérico está dividido em: genérico e transversal. A combinação dos dois cria o perfil do profissional, uma fonte de referência obrigatória.

PALAVRAS CHAVE: Animador sociocultural, competências, intervenção, profissão, educação.

[ 76 ] Ma LUISA SERRANO CAPDEVILA, JOSÉ LUIS GARCÍA LLAMAS, GLORIA PÉREZ SERRANO SIPS - PEDAGOGIA SOCIAL. REVISTA INTERUNIVERSITARIA [1139-1723 (2013) 22, 75-89] TERCERA ÉPOCA 


\section{Introducción}

Una vez más nos vemos obligados a hacer referencia a la crisis económica y social que vivimos y al reclamo de respuestas urgentes ante la globalización, la vulnerabilidad, la pérdida de marcos ideológicos y culturales y el mantenimiento del estado de bienestar.

No se puede olvidar que la animación sociocultural (ASC) está contribuyendo al desarrollo socioeducativo, así como a reforzar la identidad personal y comunitaria. Esta situación presenta puntos de inflexión que inciden directamente en los profesionales vinculados al campo social. El perfil de este profesional ha tenido que ir evolucionando, con el fin de proporcionar una respuesta adecuada a las necesidades emergentes generadas por los escenarios donde desarrolla su cometido.

En la literatura consultada se constata una cierta disociación entre la formación académica impartida por las universidades y el mundo laboral que solicita requerimientos específicos. Ante esta situación, nos hemos planteado el siguiente problema de investigación ¿Cuáles son las competencias clave que definen el quehacer profesional del animador sociocultural?

La finalidad de este trabajo consiste en identificar las exigencias que los nuevos contextos de intervención social reclaman a este agente. Se ha recogido la opinión de los profesionales en ejercicio con el objeto de determinar las competencias que desempeñan y las que necesitan mejorar. Los objetivos específicos se centran en:

- Identificar las competencias clave que pone en práctica el animador en el desarrollo de sus funciones

- Diagnosticar sus carencias formativas y

- Ofrecer sugerencias de mejora con el fin de propiciar una intervención de calidad

Este trabajo se focaliza desde la perspectiva del animador profesional, analizando las destrezas que despliega en su práctica cotidiana, pues ellos son los que, en mayor medida, pueden legitimarlas. No por ello se deja de reconocer la labor de los voluntarios y también de otros profesionales que emplean para su trabajo la metodología de la animación.

Se inicia este artículo analizando la figura del animador sociocultural y estudiando los constructos de profesión y profesionalización en el marco de la investigación. Se dedica una especial atención a las cualificaciones de este profesional. La parte empírica recoge información sobre las competencias de los animadores en ejercicio, línea en la que viene trabajando el grupo de investigación. En esta ocasión, se utiliza una metodología cualitativa y cuantitativa. Se finaliza con la presentación de los principales resultados que permiten elaborar conclusiones y propuestas de mejora.

\section{El animador}

La sociedad en la que vivimos recibe diferentes calificativos como sociedad del conocimiento, multicultural, tecnológica,... Este conjunto de apelativos demanda nuevos requerimientos a los animadores, profesión que no debe ser estática, sino adaptarse al reclamo de los tiempos. En concreto, el poder de los avances tecnológicos incide directamente en el ejercicio profesional y abre nuevos cauces a la economía, la cultura, la política, la educación, el ocio y la participación ciudadana, modificando el quehacer cotidiano de los usuarios. Esta situación exige de los animadores una formación amplia que les prepare para ser competentes en el desempeño de su profesión en sociedades versátiles y cambiantes.

La primera exigencia de cualquier profesional es tener claro el marco conceptual en el que va a desempeñar sus cometidos. Ciertamente, resulta evidente este principio, que se hace más necesario en las más complejas al estar llamadas a desempeñar sus funciones en diferentes escenarios y contextos, como es el caso que nos ocupa. A esta situación, hay que añadir que el concepto de ASC no es univoco y, por tanto, ha dado lugar a diferentes interpretaciones lo que ha dificultado su desarrollo laboral.

Creemos conveniente precisar lo que entendemos por ASC, desde una perspectiva global, que resalte los elementos esenciales de la misma. Se trata de una metodología de intervención, de carácter intencional 
y propositivo, que promueve la participación y el desarrollo de valores sociales y culturales, orientada a la promoción individual y a la transformación social, al tiempo que otorga el protagonismo a la comunidad. Para Pérez Serrano y Sarrate Capdevila (2013, p.90) "en la sociedad civil es importante impulsar la participación para avanzar en el desarrollo de la cultura democrática, por medio de prácticas sociales comprometidas que promuevan los valores subyacentes en los derechos humanos".

Ahora bien, la animación no se puede entender solo como una metodología, sino que, implica compromiso y vida. El discurso histórico con el que más se ha identificado es el que corresponde a la promoción de la participación y a su incidencia en la dinamización de la vida asociativa (Catalá, 2012).

La persona y su capacidad de iniciativa constituyen el motor fundamental que contribuye a enriquecer a los diferentes grupos sociales. De este modo, la animación trata de caracterizar, organizar y movilizar a las personas y comunidades para transformarlas en agentes activos de su propia promoción y, en la medida de lo posible, hacerlos protagonistas de su devenir.

Todo lo anterior es posible gracias al trabajo de los animadores. La animación requiere del asesoramiento o intervención de un agente cultural. Su grado de competencia está estrechamente vinculado a la calidad de su práctica profesional. Su formación, tanto inicial como continua, aparece como un factor de primera magnitud que incide directamente en la calidad de toda intervención. Nace así la necesidad de animadores con las aptitudes necesarias para dinamizar y responsabilizar a la ciudadanía de su propio progreso.

El animador es un experto que tiene como función esencial impulsar y facilitar el acceso a una vida más creativa y activa en contextos comunitarios. Dicho propósito se sustenta en el respeto y la defensa de la dignidad de la persona y en la práctica de los derechos humanos orientada hacia el estado de bienestar y la equidad social. Su misión consiste en impulsar a individuos y grupos a que espontáneamente participen en la cultura dentro del contexto en el que están inmersos, a fin de lograr los verdaderos intereses de la comunidad. De ahí que su labor se centre en ayudar y asesorar a otros a que se organicen, se planifiquen y se coordinen, dado que lo que interesa es que el proceso sea asumido por los propios participantes. En suma, el animador es la fuerza que cataliza la transformación social mediante una metodología activa y participativa orientada a promover el compromiso ciudadano.

López Noguero (2002) señala algunos rasgos del animador profesional. Entre ellos, figura, una mejor preparación y especialización que suele revertir en la calidad de los resultados, dominar los instrumentos y técnicas de intervención, recibir apoyo institucional y reconocimiento social y garantizar una mayor estabilidad de permanencia de los programas y proyectos al estar comprometidos mediante vínculo contractual.

Sus cualidades son el activo más importante para ejercer su labor. Actúa desde, con y por su personalidad singular. Sus destrezas y habilidades se basan en un saber, un saber hacer y un modo de hacer específico. No obstante, convendría delimitarlas mejor para evitar la ingerencia de otros profesionales y una posible descoordinación que dificulta, en ocasiones, el desarrollo de sus funciones.

\section{La profesionalización}

El animador sociocultural necesita afianzar su profesión. Este vocablo proviene del latín (professio,-onis) que significa: expresar, manifestar y ejercer un oficio. Según el diccionario de la Real Academia de la Lengua (2001, p.1840), la profesión es "un empleo, oficio o facultad, que alguien ejerce y por el que percibe una retribución". Desde una perspectiva descriptiva se entiende como el conjunto de funciones públicas, que la sociedad valora, desempeñadas por personas técnicamente preparadas de forma específica, que disfrutan de cierta autonomía y asumen un código deontológico común.

Estrechamente vinculado al anterior se encuentra el término profesionalización que hace referencia a la acción y efecto de profesionalizar. Significa dar carácter de profesión a una actividad. Se trata de un proceso social por el cual cualquier ocupación se llega a transformar con el tiempo en una autentica profesión consolidada. Esta secuencia implica la delimitación de las cualificaciones reconocidas por el colegio profesional correspondiente. Dichas instituciones establecen las normas, los códigos de conducta y la acreditación

[ 78 ] Ma LUISA SERRANO CAPDEVILA, JOSÉ LUIS GARCÍA LLAMAS, GLORIA PÉREZ SERRANO SIPS - PEDAGOGIA SOCIAL. REVISTA INTERUNIVERSITARIA [1139-1723 (2013) 22, 75-89] TERCERA ÉPOCA 
requerida para el ejercicio profesional. De esta forma, se delimita la diferenciación con los amateurs o aficionados. Las profesiones conllevan poder, prestigio y estatus social.

En sintonía con Sáez Carreras (2009), las profesiones se van construyendo históricamente, al tiempo que reformulan la experiencia cotidiana y resignifican la realidad social. Toda profesión depende, en buena medida, de su habilidad para mantener la creencia de que las competencias ofrecidas son necesarias a la población. En relación con lo anterior, son los propios animadores los que definen, construyen, sostienen y legitiman las competencias que es preciso mostrar para promover la creencia pública en la necesidad y pertinencia de su labor.

En la década de los setenta la animación se practicaba sin una formación específica y sistemática. Preferentemente, se ejercía con carácter voluntario, vinculada a las organizaciones religiosas y sin ánimo de lucro. Posteriormente, en los ochenta y noventa, se vio impulsada por la aparición de los ayuntamientos democráticos, que comenzaron a demandar el concurso de agentes vinculados al cambio, mejora y transformación de la sociedad. En dicha época, logró un mayor reconocimiento tanto en lo que afecta a la especificidad del curriculum como al desempeño laboral. Con el paso de los años, se ha ido afianzando la figura del animador profesional que ejerce sus funciones tanto en entidades públicas como privadas.

El estatus de esta figura presenta un carácter institucional al estar regulado por un contrato, así como por un código deontológico que delimita su comportamiento. El acceso a este puesto de trabajo suele tener carácter selectivo, basado en una cualificación reconocida, las competencias que demuestra y el compromiso con las responsabilidades que debe asumir. Al animador, como a cualquier profesional, se le exige una serie de atribuciones. Esta profesión se halla en proceso de construcción, como indican Soravilla, Pac y Cano (2011) pues aún necesita afianzar su epistemología. Además, se halla expuesta a los vaivenes de las decisiones políticas de las administraciones públicas con incidencia directa en los proyectos sociales, lo que genera inseguridad en los animadores.

Un hito significativo se produce en el año 1991, en el que se crea la carrera de Educación Social que incluye los estudios de animación sociocultural, uno de los tres grandes ámbitos de especialización, junto a la educación de adultos y la educación especializada. En el 2011, se redefinen las funciones y competencias del Técnico Superior en Animación Sociocultural reguladas en 1995, que pasa a denominarse Técnico Superior en Animación Sociocultural y Turística (Real Decreto 1684/2011) que amplia sensiblemente sus funciones y ámbitos de acción. Pertenece a la Familia Profesional de Servicios Socioculturales y a la Comunidad.

Conviene resaltar que como todas las profesiones sociales, la de animador, en estos momentos está sufriendo un punto de inflexión debido a la crisis socioeconómica existente. Se halla catalogada en la clasificación nacional de ocupaciones bajo la denominación de animador comunitario. Como tareas que debe desempeñar se encuentran todas las vinculadas a lograr un mejor disfrute del ocio y el tiempo libre, así como las relacionadas con la prevención de la marginación y la inclusión, la promoción del voluntariado y la reinserción social. Como puede observarse se insiste en la perspectiva social, si bien, conviene reseñar que la ASC también desarrolla un claro cometido de promoción cultural.

Para delimitar los entornos profesionales de este técnico, relacionados con sus ocupaciones, es imprescindible acudir a la normativa vigente ya citada. Dictamina que ejercen su labor en el sector de servicios y más en concreto en los de: desarrollo comunitario e información juvenil; servicios culturales (de lectura e información, de tecnologías de la información y la comunicación y de recursos y productos culturales diversos); servicios turísticos y en el de ocio y tiempo libre (de actividades lúdicas y deportivas, medioambientales y de aire libre).

\section{Competencias}

En la actualidad, ninguna profesión puede presuponer los conocimientos, habilidades y destrezas que se pueden requerir en un futuro próximo. Por ello, la inquietud por la formación continua es una exigencia imprescindible que incide directamente en la selección de buenos profesionales. 
Se puede constatar que no existe unanimidad en la delimitación conceptual de lo que se entiende por el constructo competencia, dada su afinidad a otros conceptos como, conocimientos, capacidades, habilidades y destrezas. Con el fin de realizar una primera aproximación al tema se han consultado diferentes fuentes (Alonso, 2009; Escudero Muñoz, 2009 y MEC, 2006; entre otros).

Desde una perspectiva etimológica el sustantivo competencia procede del verbo latino competere, y de éste se deriva el adjetivo competens-tis que significa competente, apropiado, conveniente y apto. En términos generales, este vocablo se otorga al buen conocedor de una técnica, disciplina o arte.

En general, se entiende de formas diversas, si bien, todas ellas, coinciden en un aspecto esencial: el saber hacer, sin descuidar el conocer y el saber ser. En este sentido, el MEC (2006), refiriéndose a la acepción académica y no a la de atribución profesional, establece que:

“las competencias son una combinación de conocimientos, habilidades (intelectuales, manuales, sociales, etc.), actitudes y valores que capacitarán a un titulado para afrontar con garantías la resolución de problemas o la intervención en un asunto, en un contexto académico, profesional o social determinado". (p.6)

Completando la anterior perspectiva, desde el punto de vista laboral, dicho término hace alusión a la capacidad para desempeñar las tareas inherentes a un empleo concreto, para el que se tienen en cuenta el nivel y la especialización. Se refiere a un conjunto de actividades que está llamado a desempeñar de forma autónoma cualquier profesional. Deben interpretarse desde un enfoque sistémico, como acciones integradas sustentadas en un proyecto ético que contribuyan a solucionar los problemas.

De las distintas definiciones analizadas se desprende que las competencias deben reunir una serie de requisitos, como su carácter aplicable o transferible a situaciones diversas, así como al logro de objetivos diversificados. Para Sáez Carreras (2009) las distintas definiciones integran y orquestan recursos que se van adquiriendo por formación, por experiencia y mediante contacto directo con la práctica. Se ponen de manifiesto al actuar con pertinencia en una situación compleja y armonizan conocimientos, aptitudes y acciones. Las competencias clave, denominadas también fundamentales y/o básicas, han de proporcionar un soporte sólido que posibilite la formación a lo largo de la vida.

En general, en las distintas aproximaciones a este concepto complejo, aparecen dos aspectos fundamentales: su vinculación a la "utilidad" en sentido amplio, asociada a intereses sociales, económicos y culturales y, por otro lado, la facultad de ser "adquiribles" por los individuos (Alonso, 2009).

El Espacio Europeo de Educación Superior, a través del Libro Blanco (2005) examina las competencias que se refieren al animador sociocultural. El estudio comparado, realizado entre titulados, asociaciones y profesionales, coincide en clasificarlas en transversales o genéricas -necesarias para el desempeño de cualquier profesión-y específicas -que engloban las exigencias concretas que requiere el ejercicio de una determinada profesión-. Las primeras comprenden aspectos relacionados con la organización y planificación. Las segundas, hacen referencia al saber, saber hacer y saber ser.

Las competencias que definen este perfil profesional aparecen en la legislación promulgada en el 2011, que reseña las siguientes: "programar, organizar, implementar y evaluar intervenciones de animación sociocultural y turística, promoviendo la participación activa de las personas y grupos destinatarios, y coordinando las actuaciones de los profesionales y voluntarios". Se completan con las de índole profesional, personal y social y las respectivas cualificaciones profesionales.

Los desafíos que permean todas las competencias que requiere el animador están vinculados con la capacidad de innovación. Debe ser capaz de sobrepasar los límites y fronteras del conocimiento y destrezas adquiridas. Entre dichos retos se pueden mencionar, el espíritu emprendedor, la responsabilidad, la versatilidad y la creatividad. Estos agentes deben ser capaces de mirar la realidad y perforar sus velos con el fin de adelantarse y contribuir a la prevención de problemas sociales.

[ 80 ] Ma LUISA SERRANO CAPDEVILA, JOSÉ LUIS GARCíA LLAMAS, GLORIA PÉREZ SERRANO SIPS - PEDAGOGIA SOCIAL. REVISTA INTERUNIVERSITARIA [1139-1723 (2013) 22, 75-89] TERCERA ÉPOCA 


\section{Metodología}

Para la identificación de las competencias del animador y el diagnóstico de sus principales carencias formativas, objetivos específicos planteados en la investigación, la información recogida se han estructurado en tres grandes categorías. Éstas se basan en la literatura existente sobre el tema, así como en los datos aportados por los animadores en ejercicio. Se las denomina

- Metodológicas: recogen los principales elementos que incumben directamente a la intervención sociocultural. En este sentido, cabe citar las habilidades para el trabajo en equipo, la capacidad para afrontar y solucionar problemas derivados de su actividad, el dominio de los métodos de trabajo en equipo y el desarrollo de procesos de dinamización de la participación.

- De planificación: asociadas a aspectos como la coordinación, ejecución y evaluación de proyectos, la coordinación entre los diferentes agentes sociales, así como la comunicación en el desarrollo y participación en las distintas dimensiones de la actividad profesional.

- Genéricas: también llamadas básicas pues hacen alusión a aquellos dominios que debe poseer cualquier profesional para el desempeño de su labor. Entre ellos, se han seleccionado los más vinculados con los cometidos que desarrolla el animador como: la utilización de las TICs, analizar y comprender los contextos sociales, la localización de recursos, la participación en equipos interdisciplinares, detectar necesidades de intervención en los grupos y el compromiso ético.

En el proceso de investigación empírica uno de los momentos importantes consiste en la selección y elaboración de instrumentos de recogida de datos. Dado el enfoque del trabajo, se optó por realizar entrevistas con informantes clave y la elaboración de un cuestionario diseñado específicamente para esta ocasión. Tiene por objeto recoger información valiosa de los protagonistas, es decir, los animadores. Se estructuró en dos grandes apartados: el primero recoge aspectos relativos a las características personales, contextuales, académicas y laborales; mientras que el segundo presenta las competencias básicas de este profesional. Se concluyó con dos cuestiones abiertas. Se remitió vía "on-line" a toda la población objeto de estudio configurada por 530 animadores. Se recibieron debidamente cumplimentados 376, lo que eleva el nivel de respuesta al 70,94\%, participación significativa sin duda influida por la reiteración de mensajes motivadores.

En lo que se refiere al análisis de las características técnicas del cuestionario se determinó la fiabilidad del instrumento mediante el procedimiento alpha de Cronbach que ofreció un valor de 0,930, lo que indica un nivel elevado de fiabilidad. Respecto a la validez de contenido se ha recurrido a la opinión de jueces y expertos vinculados al campo laboral de la animación, siendo dos de ellos especialistas en metodología de investigación. Los resultados fueron satisfactorios, tanto en lo que respecta a la organización del cuestionario como a la claridad expresiva, si bien se incorporaron algunas mejoras en la redacción de los ítems.

Para llevar a cabo el tratamiento estadístico de los datos en su vertiente cuantitativa, se recurrió al programa estadístico SPSS en su versión 18. De esta forma se realizaron estudios de carácter descriptivo dirigidos a conocer de forma más precisa las características personales de los sujetos de la muestra, a través de porcentajes y valores medios en la calificación de las competencias contempladas.

En un segundo momento, se utilizó la prueba del ANOVA para comprobar la existencia o no de diferencias significativas entre los grupos de análisis. En concreto, se llevó a cabo sobre los cuatro grupos de edad previamente configurados. También, se empleó la prueba para comprobar la igualdad de medias de WELCH; así como los contrastes "a posteriori" entre aquellos grupos cuyo valor de " $F$ " alcanzasen los niveles de confianza mínimos fijados en el 95\%. Por último, se considera de especial interés comprobar, mediante el Análisis Factorial Confirmatorio (AFC) la agrupación de las competencias en torno a una serie de constructos o factores, con el fin de estudiar la posible correspondencia con el marco teórico. 


\section{Descripción de resultados}

Los datos de la muestra revelan que predomina el sexo femenino con un 70,1\%; lo que viene a confirmar las conclusiones de estudios anteriores, si bien se constata un incremento paulatino de varones. El tramo de edad con mayor presencia en la muestra se encuentra entre los $31-40$ años con un $43,7 \%$ de los sujetos. El nivel educativo es bastante elevado: un $60,9 \%$ ha realizado estudios universitarios y un $39,1 \%$ posee una titulación media identificada con bachillerato, formación profesional y acceso a la universidad. Además, la actividad profesional básica se centra en dos ámbitos: la población juvenil con un 36,0\% y los adultos y mayores con un 32,2\%. La anterior información desvela la importancia actual del animador en dichos colectivos caracterizados por diferentes situaciones de riesgo y exclusión social. Al mismo tiempo, estos agentes consideran que deberían estar más presentes en escenarios tales como: el educativo, intercultural, cívico-social, de ocio y lugares de acogida de grupos en situaciones de vulnerabilidad.

Las competencias se contemplan desde dos dimensiones: las que desempeñan en su actividad profesional y las que necesitan mejorar. En la siguiente tabla se recogen los resultados aportados por la muestra. Se han ordenado de mayor a menor, teniendo en cuenta los porcentajes de respuesta positiva de los profesionales. En paralelo, se muestran los porcentajes de mejora solicitados.

Tabla 1. Desempeño de las competencias profesionales y necesidad de mejora

\section{Competencias}

Desempeño (\%) Necesito mejorar (\%)

\begin{tabular}{lll}
\hline Dinamizar y movilizar a los participantes & 97,1 & 52,4 \\
\hline Capacidad para la resolución de problemas & 90,6 & 48,2 \\
\hline Desarrollar habilidades de trabajo en equipo & 85,7 & 45,2 \\
\hline Desarrollar actitudes éticas & 84,3 & 37,6 \\
\hline $\begin{array}{l}\text { Comunicarse de forma oral y escrita en todas las dimensiones } \\
\text { de su actividad profesional }\end{array}$ & 82,8 & 47,7 \\
\hline Dominar las metodologías y técnicas de trabajo en grupo & 81,8 & 50,0 \\
\hline Participar en un equipo interdisciplinar & 79,4 & 48,8 \\
\hline Coordinar a los diferentes agentes implicados en los proyectos & 70,7 & 52,9 \\
\hline Dirigir, ejecutar y evaluar proyectos y acciones de intervención & 70,6 & 42,4 \\
\hline Utilizar de forma eficaz las TIC & 63,9 & 46,3 \\
\hline Localizar y generar recursos para el desarrollo de la intervención & 62,1 & 54,1 \\
\hline Analizar y comprender los contextos sociales & 60,6 & 60,0 \\
\hline Detectar las necesidades de intervención en un grupo con & 58,9 & $50,6 \%$ \\
\hline características específicas & & \\
\hline
\end{tabular}

En líneas generales, los animadores aseguran que realizan las mencionadas competencias en un alto grado. Así, por encima del $90 \%$ ejercen dos tareas importantes en su actividad laboral: "dinamizar y movilizar a los participantes" y "tener capacidad para resolver problemas". Entre el 80-90\% nos encontramos con destrezas como "desarrollar habilidades de trabajo en equipo", "desarrollar actitudes éticas", "comunicarse de forma oral y escrita en su actividad profesional" y "dominar las metodologías y técnicas de trabajo en grupo". En suma, sobresale la importancia que en el ejercicio profesional tiene el trabajo en grupo, pues valoran, de forma muy positiva, tanto su desarrollo como el dominio técnico y metodológico que esta tarea lleva asociada. En el mismo nivel se encuentran los compromisos éticos derivados de las exigencias de una profesión de índole social.

[ 82 ] Ma LUISA SERRANO CAPDEVILA, JOSÉ LUIS GARCÍA LLAMAS, GLORIA PÉREZ SERRANO SIPS - PEDAGOGIA SOCIAL. REVISTA INTERUNIVERSITARIA [1139-1723 (2013) 22, 75-89] TERCERA ÉPOCA 
Un tercer grupo, situado entre el 70-80\%, viene configurado por las siguientes competencias: "participar en un equipo interdisciplinar", "coordinar a los diferentes agentes implicados en los proyectos" y "dirigir, ejecutar y evaluar proyectos y acciones de intervención". Como puede constatarse, la mayoría de ellas se vinculan al campo técnico, pues requieren de un dominio de estos contenidos en su formación inicial y permanente.

En un cuarto grupo, entre 60-70\%, se sitúan aspectos como: "utilizar de forma eficaz las TICs"; "localizar y generar recursos para el desarrollo de la intervención" y "analizar y comprender los contextos sociales". Estas competencias se asocian al ámbito de los medios y recursos que es preciso conocer y utilizar para desempeñar de forma eficaz la tarea profesional. Por último, la menos valorada es la que hace referencia a "la detención de necesidades de intervención en un grupo de características específicas", aunque alcanza el $58,9 \%$.

En lo que se refiere a las competencias que necesitan mejorar solamente "analizar y comprender los contextos sociales" alcanza un 60\%. Sin duda, subyace en este resultado la necesidad de profundizar en el elemento básico que debe sustentar toda intervención. Por encima del 50\% se sitúan: "localizar y generar recursos para la intervención", "coordinar a los diferentes agentes implicados en los proyectos", "dinamizar y movilizar a los participantes", "detectar necesidades de intervención en grupos con características específicas" y "dominar las metodologías y técnicas de trabajo en grupo". En este bloque se entremezclan aspectos del desarrollo diario de la profesión con cuestiones de tipo técnico que probablemente requieren de una preparación más cualificada.

Superando el $40 \%$ en la demanda de mejora de las competencias nos encontramos con "participar en un equipo interdisciplinar", "capacidad para la resolución de problemas", "comunicarse de forma oral y escrita en la actividad profesional", "utilizar de forma eficaz las TIC", "desarrollar habilidades de trabajo en equipo" y "dirigir, ejecutar y evaluar proyectos y acciones de intervención". En todas ellas subyace la demanda de una formación permanente. De hecho, declaran que necesitan prepararse mejor para llevar a cabo tareas de trabajo en grupo y estar al día en el uso de las tecnologías de la información y la comunicación, tan cambiantes en el mundo actual. Les preocupa, igualmente, el diseño y evaluación de los proyectos de intervención en el contexto social.

Tan solo "desarrollar actitudes éticas" presenta una demanda menor. Concretamente un 37,6\% pide una mejora en esta competencia vinculada al respecto al individuo que es objeto de la intervención del profesional.

En síntesis, debemos reseñar que algunas de las competencias desarrolladas con mayor asiduidad en la tarea profesional, no necesariamente coinciden con las demandas de mejora de las mismas. De hecho, los porcentajes más elevados se vinculan a diversas cuestiones de tipo técnico y especializado, mientras que las asociadas a la práctica cotidiana precisan de un nivel de mejora inferior posiblemente generado por la experiencia adquirida.

Creemos que una de las variables importantes de categorización es la edad de los animadores. Debido a la dispersión de los datos, se han clasificado en cuatro grupos (20-30; 31-40; 41-50 y más de 51 años). En la tabla siguiente se presentan los valores medios de cada una de las competencias, tanto de forma global como de cada grupo de edad. Además, se ha añadido el valor del estadístico "F" del ANOVA, así como la significatividad del mismo, que permite constatar la existencia de diferencias significativas entre los grupos de edad. Estos análisis se realizan sobre las competencias que desempeñan los profesionales en su campo de trabajo. La escala de valoración oscila entre 1 y 10.

En la tabla 2 hemos podido comprobar la existencia de diferencias altamente significativas en alguna de las competencias. Así, el valor del estadístico de contraste " $F$ " es más elevado en la utilización eficaz de las TICs, donde el grupo de mayor edad es el que tiene una opinión más positiva; mientras que el colectivo entre 41 y 50 años es el que ofrece una valoración más baja y crítica. También tienen un nivel bajo el grupo de menor edad. Los contrastes "a posteriori" constatan la significatividad de esas diferencias, como se puede comprobar en el gráfico 1. 
Tabla 2. Edad de los profesionales y valoración de las competencias desempeñadas

\begin{tabular}{|c|c|c|c|c|c|c|c|}
\hline Competencias & Global & $20-30$ & $31-40$ & $41-50$ & Más 51 & "F" & $\begin{array}{l}\text { Signifi- } \\
\text { catividad }\end{array}$ \\
\hline Analizar y comprender contextos & 7,43 & 7,44 & 7,38 & 7,12 & 8,29 & 1,384 & 0,248 \\
\hline Dominar metodologías y técnicas de trabajo grupo & 8,07 & 8,14 & 7,97 & 7,88 & 8,50 & 0,826 & 0,481 \\
\hline Dinamizar y movilizar a los participantes & 8,89 & 9,33 & 8,25 & 9,08 & 9,43 & 13,844 & 0,000 \\
\hline Coordinar a los agentes implicados en proyectos & 7,50 & 7,32 & 7,95 & 6,48 & 9,14 & 8,201 & 0,000 \\
\hline Detectar necesidades de intervención en grupo & 7,44 & 7,41 & 7,26 & 7,28 & 8,86 & 3,336 & $\mathrm{O}, \mathrm{O} 2 \mathrm{O}$ \\
\hline Dirigir, ejecutar y evaluar proyectos & 7,78 & 7,37 & 8,19 & 7,55 & 8,21 & 2,528 & 0,058 \\
\hline Localizar y generar recursos para intervenir & 7,20 & 7,21 & 7,67 & 5,92 & 8,07 & 6,834 & 0,000 \\
\hline Desarrollar habilidades de trabajo en equipo & 8,38 & 8,63 & 7,83 & 8,70 & 8,86 & 8,843 & $0, \bigcirc \circ \bigcirc$ \\
\hline Participar en un equipo interdisciplinar & 7,94 & 8,66 & 7,65 & 7,38 & 8,43 & 7,455 & 0,000 \\
\hline Capacidad para resolución de problemas & 8,75 & 8,76 & 8,60 & 8,72 & 9,71 & 3,166 & 0,025 \\
\hline Utilizar de forma eficaz las TIC & 7,25 & 6,87 & 7,93 & 6,05 & 9,29 & 15,931 & 0,000 \\
\hline Comunicarse de forma oral y escrita & 8,09 & 8,05 & 8,51 & 7,22 & 9,14 & 7,058 & 0,000 \\
\hline Desarrollar actitudes éticas & 8,50 & 8,85 & 8,56 & 7,70 & 8,79 & 5,578 & 0,001 \\
\hline
\end{tabular}

Gráfico 1. Desempeña. Utilizar de forma eficaz las TIC

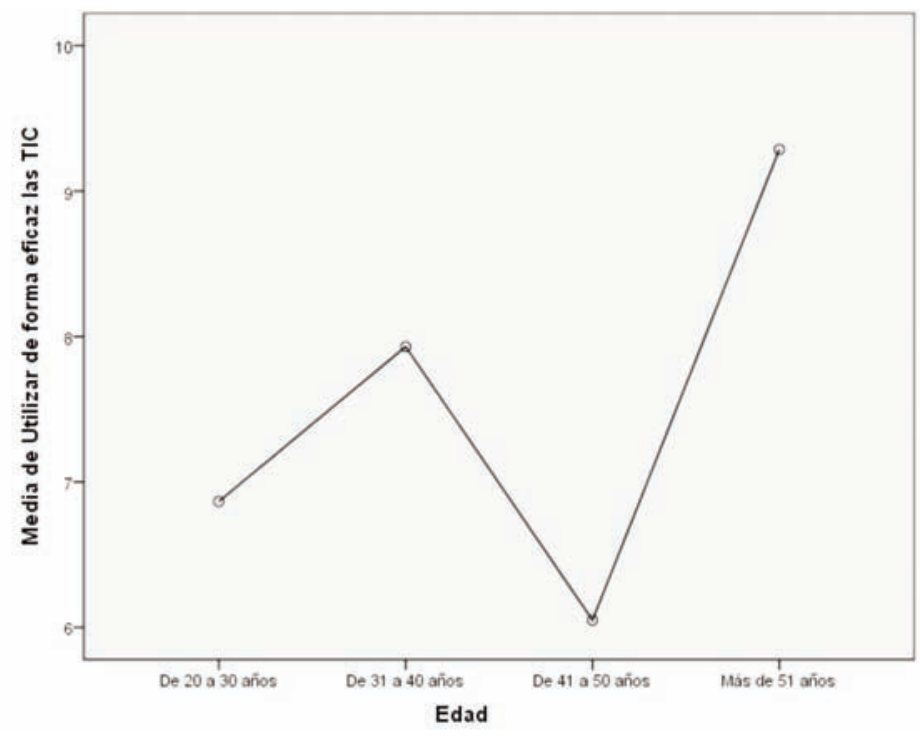

También se manifiestan diferencias significativas elevadas ( $F=13,844)$ en la competencia de "dinamizar y movilizar a los participantes" en los grupos extremos, mientras que los más críticos se corresponden con el grupo de edad entre 31 y 40 años.

"Desarrollar habilidades de trabajo en equipo" $(F=8,843)$ pone de relieve la existencia de diferencias significativas entre los dos grupos de mayor edad y los otros dos, sobre todo con el de 31-40 años. Valores similares se encuentran en la competencia de "coordinar a los agentes implicados en los proyectos" si bien, en este caso, el valor más bajo se da en el grupo entre 41 y 50 años, lo que demuestra la existencia de diferencias significativas con los demás.

La "participación en un equipo interdisciplinar", con F=7,455, presenta una diferencia significativa entre los más jóvenes y los más veteranos. La competencia asociada a la "comunicación fluida de forma oral y escrita", mantiene unos valores similares. El grupo más crítico es el de 41-50 años. 
Un comportamiento más homogéneo se observa entre los distintos grupos de edad, en competencias tales como: "localizar y generar recursos para intervenir en los contextos sociales" y "desarrollar actitudes éticas" en las que el grupo edad 41-50 difiere del resto al ser el más bajo. A un nivel de confianza menor (95\%), se aprecian diferencias significativas entre los grupos en competencias como "detectar necesidades de intervención en grupo" y "la resolución de problemas", donde destaca el colectivo de mayor edad.

Es importante resaltar que en las competencias que definen la identidad de la acción profesional del animador, no se aprecian diferencias acusadas entre los grupos. Esto viene a confirmar que forman parte de la práctica cotidiana de la animación. En concreto, se refieren a: "analizar y comprender los contextos" y a "dominar metodologías y técnicas de trabajo grupo". Obtiene un valor próximo a las anteriores, la función de "dirigir, ejecutar y evaluar proyectos", donde el sector más joven ofrece una valoración menor.

Para completar el apartado anterior, a continuación, se estudian las necesidades de mejora de los animadores que quedan reflejadas en el siguiente cuadro:

Tabla 3. Edad de los profesionales y necesidades de mejora de las competencias

\begin{tabular}{|c|c|c|c|c|c|c|c|}
\hline Competencias & Global & $20-30$ & $31-40$ & $41-50$ & Más 51 & "F" & $\begin{array}{c}\text { Signifi- } \\
\text { catividad }\end{array}$ \\
\hline Analizar y comprender contextos & 6,77 & 6,44 & 6,90 & 6,97 & 5,79 & 1,627 & 0,183 \\
\hline Dominar metodologías y técnicas de trabajo grupo & 6,86 & 6,17 & 7,32 & 7,00 & 5,93 & 4,064 & 0,007 \\
\hline Dinamizar y movilizar a los participantes & 6,55 & 5,13 & 7,54 & 6,83 & 5,79 & 16,065 & 0,000 \\
\hline Coordinar a los agentes implicados en proyectos & 6,51 & 6,39 & 6,79 & 6,01 & 5,86 & 1,313 & 0,270 \\
\hline Detectar necesidades de intervención en grupo & 6,46 & 6,61 & 6,27 & 6,28 & 5,93 & 0,505 & 0,679 \\
\hline Dirigir, ejecutar y evaluar proyectos & 6,23 & 6,05 & 5,96 & 6,67 & 5,86 & 1,094 & 0,352 \\
\hline Localizar y generar recursos para intervenir & 6,53 & 6,58 & 6,46 & 6,33 & 5,93 & 0,251 & 0,861 \\
\hline Desarrollar habilidades de trabajo en equipo & 6,20 & 5,50 & 6,56 & 6,42 & 5,50 & 3,040 & 0,029 \\
\hline Participar en un equipo interdisciplinar & 6,05 & 5,20 & 6,50 & 6,70 & 5,36 & 5,979 & 0,001 \\
\hline Capacidad para resolución de problemas & 6,13 & 5,54 & 6,46 & 6,35 & 5,07 & 2,566 & 0,055 \\
\hline Utilizar de forma eficaz las TICs & 6,12 & 5,78 & 6,50 & 5,58 & 5,86 & 2,194 & 0,089 \\
\hline Comunicarse de forma oral y escrita & 5,77 & 5,21 & 5,75 & 6,30 & 5,64 & 2,209 & 0,087 \\
\hline Desarrollar actitudes éticas & 5,46 & 5,18 & 5,54 & 5,25 & 5,36 & 0,348 & 0,791 \\
\hline
\end{tabular}

Gráfico 2. Necesita mejorar. Participar en un equipo interdisciplinar

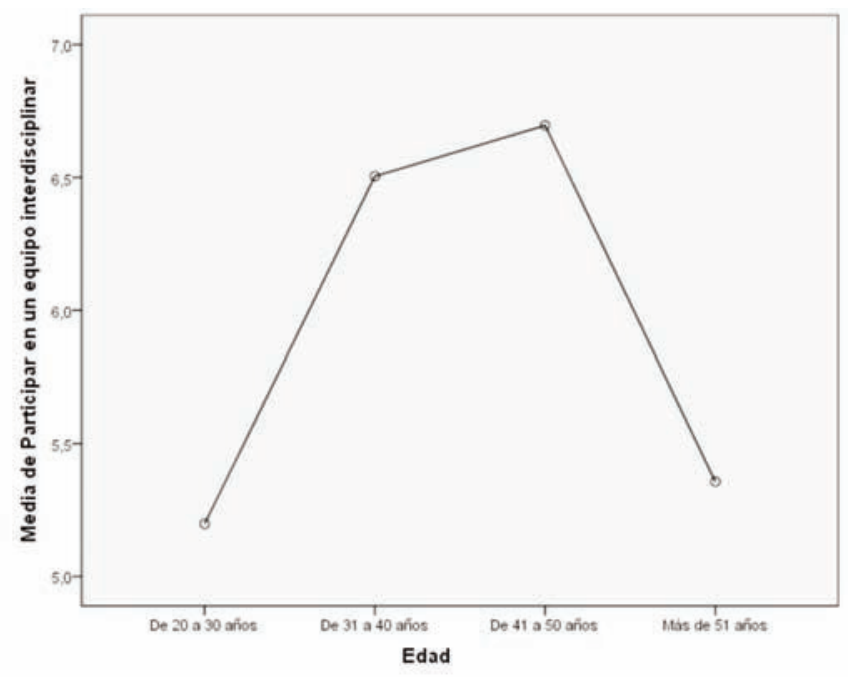


A diferencia de lo que ocurría anteriormente, al preguntarles sobre las mismas competencias y la necesidad de mejorarlas, nos encontramos con pocas diferencias significativas. Las más elevadas afectan a la función de "dinamizar y movilizar a los participantes" con fuerte demanda en el grupo de edad entre 31 y 40 años y la más baja en los de menor edad; quizá motivada por los cambios producidos en la formación inicial de estos profesionales.

Con un valor de F=5,979 aparece la necesidad de "mejorar la participación en un equipo interdisciplinar", con mayor demanda por parte de los grupos de edad intermedia. Los contrastes de medias ponen de relieve la existencia de diferencias significativas entre el grupo más joven y los de edad intermedia, no así con el grupo de los más veteranos, tal y como se puede comprobar en el gráfico 2.

Las diferencias se elevan a un nivel de confianza del $99 \%$ en la "necesidad de mejorar el dominio de las metodologías y técnicas de trabajo en grupo", más demandada por los animadores de edad intermedia. Nos encontramos con el límite del 95\% en cuanto a "desarrollar habilidades de trabajo en grupo" y a la "capacidad para resolver problemas".

Ligeras diferencias, pero no significativas al nivel fijado, se manifiestan en cometidos como: "mejora de la comunicación oral y escrita" y la "utilización de las TICs". En el resto de las competencias contempladas, el nivel de demanda es muy similar en todas las edades, lo que parece reconfirmar el deseo de perfeccionamiento profesional de todo animador.

Se aplicó el análisis factorial al desempeño de las competencias profesionales para valorar su agrupación en torno a las funciones más significativas desarrolladas por los animadores. Así, se puede constatar que la varianza total explicada alcanza un porcentaje del 79,572\% en torno a cuatro factores, que se vuelven a confirmar cuando se repite la rotación de factores, como se puede constatar en los cuadros siguientes.

Tabla 4. Varianza total explicada

\begin{tabular}{|c|c|c|c|c|c|c|c|c|c|}
\hline \multirow[t]{2}{*}{ Componente } & \multicolumn{3}{|c|}{ Autovalores iniciales } & \multicolumn{3}{|c|}{$\begin{array}{l}\text { Sumas de las saturaciones } \\
\text { al cuadrado de la extracción }\end{array}$} & \multicolumn{3}{|c|}{$\begin{array}{l}\text { Suma de las saturaciones } \\
\text { al cuadrado de la rotación }\end{array}$} \\
\hline & Total & $\begin{array}{l}\text { \% de la } \\
\text { varianza }\end{array}$ & $\begin{array}{c}\% \\
\text { acumulado }\end{array}$ & Total & $\begin{array}{l}\text { \% de la } \\
\text { varianza }\end{array}$ & $\begin{array}{c}\% \\
\text { cumulado }\end{array}$ & Total & $\begin{array}{c}\text { \% de la } \\
\text { varianza }\end{array}$ & $\begin{array}{c}\% \\
\text { acumulado }\end{array}$ \\
\hline 1 & 5,764 & 44,337 & 44,337 & 5,764 & 44,337 & 44,337 & 2,860 & 22,000 & 22,000 \\
\hline 2 & 2,292 & 17,633 & 61,970 & 2,292 & 17,633 & 61,970 & 2,844 & 21,878 & 43,878 \\
\hline 3 & 1,270 & 9,768 & 71,738 & 1,270 & 9,768 & 71,738 & 2,399 & 18,452 & 62,330 \\
\hline 4 & 1,018 & 7,834 & 79,572 & 1,018 & 7,834 & 79,572 & 2,242 & 17,242 & 79,572 \\
\hline 5 & 671 & 5,160 & 84,732 & & & & & & \\
\hline 6 & ,495 & 3,805 & 88,538 & & & & & & \\
\hline 7 & ,353 & 2,719 & 91,257 & & & & & & \\
\hline 8 & ,324 & 2,493 & 93,749 & & & & & & \\
\hline 9 & ,276 & 2,120 & 95,869 & & & & & & \\
\hline 10 & , 190 & 1,461 & 97,330 & & & & & & \\
\hline 11 & , 167 & 1,288 & 98,618 & & & & & & \\
\hline 12 & ,121 & ,930 & 99,548 & & & & & & \\
\hline 13 & ,059 & 452 & 100,000 & & & & & & \\
\hline
\end{tabular}

Método de extracción: Análisis de Componentes principales.

Seguidamente se presenta la matriz de componentes rotados mediante el método: Normalización Varimax con Kaiser.

[ 86 ] Ma LUISA SERRANO CAPDEVILA, JOSÉ LUIS GARCÍA LLAMAS, GLORIA PÉREZ SERRANO SIPS - PEDAGOGIA SOCIAL. REVISTA INTERUNIVERSITARIA [1139-1723 (2013) 22, 75-89] TERCERA ÉPOCA 
Tabla 5. Matriz de componentes rotados

\begin{tabular}{|c|c|c|c|c|}
\hline & \multicolumn{4}{|c|}{ Componente } \\
\hline & 1 & 2 & 3 & 4 \\
\hline DESEMPEÑAS: Analizar y comprender contextos &, 262 &, 078 & ,619 & ,351 \\
\hline Dominar las metodologías y técnicas de trabajo en grupo &, 190 &, 838 &,- 019 & ,188 \\
\hline Dinamizar y movilizar a los participantes &,- 296 & 641 & 379 & ,221 \\
\hline Coordinar a los diferentes agentes implicados en los proyectos &, 732 & ,129 & ,102 &, 552 \\
\hline $\begin{array}{l}\text { Detectar las necesidades de intervención en un grupo con } \\
\text { características específicas }\end{array}$ & ,471 &, 351 &, 376 &, 558 \\
\hline Dirigir, ejecutar y evaluar proyectos y acciones de intervención &, 864 &, 035 &, 106 & ,227 \\
\hline Localizar y generar recursos para el desarrollo de la intervención &, 530 & ,137 & 390 &, 588 \\
\hline Desarrollar habilidades de trabajo en equipo &,- 051 &, 882 & ,059 & ,०57 \\
\hline Participar en un equipo interdisciplinar &,- O53 & ,294 & ,718 & ,482 \\
\hline Capacidad para la resolución de problemas &, 301 &, 828 &, 075 &,- 033 \\
\hline Utilizar de forma eficaz las TIC & ,215 &, 069 &, 079 &, 878 \\
\hline Comunicarse de forma oral y escrita en su actividad profesional & ,796 &, 102 &, 520 &,$\bigcirc 71$ \\
\hline Desarrollar actitudes éticas &, 329 &,$- \mathrm{O} 21$ &, 870 &,- 090 \\
\hline
\end{tabular}

Método de extracción: Análisis de componentes principales.

Método de rotación: Normalización Varimax con Kaiser.

a. La rotación ha convergido en 7 iteraciones.

Puesto que la varianza explicada a partir de los cuatro componentes principales, según tabla 4, se eleva al 79,572\%, el propio sistema elimina el resto. Como técnica de reducción de la información es importante elegir de forma adecuada el punto de corte para posteriores análisis referidos a la saturación de los cuatro factores. En la tabla 5 se presentan los valores de la correlación de cada una de las competencias con los componentes rotados, que permite, mediante la rotación VARIMAX, seleccionar y agrupar los valores más elevados de la correlación.

El factor uno agrupa las competencias relacionadas con: "dirigir, ejecutar y evaluar proyectos y acciones de intervención", "comunicarse de forma oral y escrita en todas las dimensiones de su actividad profesional" y "coordinar a los diferentes agentes implicados en los proyectos". Por lo tanto, se podría identificar con las competencias derivadas de la planificación.

Dentro del factor dos aparecen las competencias vinculadas con: "el desarrollo de habilidades para el trabajo en equipo", "dominar las metodologías y técnicas de trabajo en grupo", "la capacidad para la resolución de problemas" y "dinamizar y movilizar a los participantes". Nos estamos refiriendo a la forma de trabajo de un profesional que desempeña una serie de funciones de carácter metodológico.

El tercer factor se satura en tres competencias: "el desarrollo de actitudes éticas", "la participación en un equipo interdisciplinar" y "analizar y comprender los contextos sociales". Se pueden identificar con las competencias genéricas que ayudan al profesional a desarrollar de forma eficaz y comprometida su tarea.

En el cuarto factor nos encontramos con "la utilización eficaz de las TIC" y, en menor medida, "localizar y generar recursos para el desarrollo de la intervención social" y "detectar necesidades de intervención en un grupo con características específicas". De alguna forma serían competencias transversales vinculadas a la tarea profesional del animador.

En suma, el análisis factorial revela que las competencias ejercidas por los profesionales se agrupan en cuatro campos de especialización que guardan relación con las propuestas teóricas previas, en líneas generales, aunque se añade una nueva, puesto que las competencias genéricas se dividen en dos: las genéricas propiamente dichas y las trasversales. 


\section{Conclusiones y propuestas}

Con carácter general, se puede afirmar que nos encontramos ante un colectivo inquieto, responsable e interesado en conseguir mejores niveles de calidad en el desempeño de su profesión. Se ha podido observar que ponen en práctica en su vida laboral las competencias estudiadas. Ahora bien, esto no impide el deseo de seguir formándose.

Los resultados de la investigación evidencian que los animadores suelen desempeñar en un alto grado las competencias analizadas. Entre ellas sobresalen dos, importantes en su práctica profesional: "dinamizar y movilizar a los participantes" y "tener capacidad para resolver problemas". En cuanto a las demandas de perfeccionamiento, coinciden bastante con las que desempeñan, esto puede deberse a que son conscientes de que siempre se puede mejorar el trabajo realizado. En este sentido, declaran que precisan mejorar: "analizar y comprender los contextos sociales" " "coordinar a los diferentes agentes implicados en los proyectos"

La edad es un factor importante que incide directamente en el desempeño de las competencias profesionales ya que aportan diferencias significativas según los grupos establecidos. No obstante, existen algunas en las que no se registran diferencias acusadas entre grupos, tales como: "analizar y comprender los contextos" y "dominar metodologías y técnicas de trabajo en grupo". Estos resultados vienen a confirmar las funciones que ejercen en su práctica cotidiana y definen la identidad de la acción profesional del animador.

La investigación ha puesto de manifiesto que los animadores de edad intermedia son los que más necesitan mejorar. Esta situación es comprensible, dado que los más jóvenes han tenido la oportunidad de cursar titulación universitaria y, los más mayores, ya cuenta con una dilatada experiencia laboral.

El análisis factorial confirmatorio ha permitido constatar que la clasificación inicial de tres campos vinculados a la actividad profesional, se ha transformado en cuatro en el trabajo empírico. Así, existe una buena identificación con las competencias de planificación y metodológicas. En cambio, la categoría de las genéricas, se ha desglosado en dos: la que se identifica con dicha denominación y una nueva llamada competencia transversal. Los resultados revelan que quedarían definidas, por lo tanto, en competencias de: planificación, metodológicas, genéricas y transversales. Todas ellas propias de los profesionales del ámbito sociocultural.

Por otro lado, del estudio cualitativo se desprende la preocupación de los animadores socioculturales por lograr una mayor visibilidad profesional y un estatus reconocido en la sociedad. Al mismo tiempo, son conscientes que en los momentos de crisis económica los trabajos vinculados con el bienestar social experimentan un proceso de inflexión.

Los resultados de este estudio, sustentados en la práctica del animador sociocultural, aportan sugerencias de gran interés que permiten generar propuestas de formación en conexión con el ejercicio profesional.

Del trabajo realizado se desprende que son los profesionales, en contacto con la práctica diaria, una fuente de obligada consulta, para definir las competencias que precisan para el ejercicio de sus funciones. La formación teórica enriquecida con la práctica cotidiana está contribuyendo a la legitimidad de una profesión que se va abriendo camino en contextos complejos, lo que exige a los animadores una redefinición constante de sus funciones.

\section{Referencias bibliográficas}

Agencia Nacional de Evaluación de la Calidad y Acreditación. (2005). Libro Blanco. Título de Grado en Pedagogía y Educación Social. Madrid: ANECA.

Alonso, L. E. et al. (2009). El debate de las competencias. Una investigación cualitativa sobre el espacio superior y el mercado de trabajo en España. Madrid: ANECA.

Catalá Gorgues, R. (2012). El marco de la formación profesional y su incidencia en el campo de la animación sociocultural: nuevas figuras profesionales. Quaderns d'Animació i Educació Social, no 15 enero de 2012. Recuperado de: http://quadernsanimacio.net

[ 88 ] Ma LUISA SERRANO CAPDEVILA, JOSÉ LUIS GARCíA LLAMAS, GLORIA PÉREZ SERRANO SIPS - PEDAGOGIA SOCIAL. REVISTA INTERUNIVERSITARIA [1139-1723 (2013) 22, 75-89] TERCERA ÉPOCA 
Escudero Muñoz, J. M. (2009). Las competencias profesionales y la formación universitaria: posibilidades y riesgos. Pedagogía Social. Revista Interuniversitaria, 16, pp. 65-84.

López Noguero, F. (2002). El perfil del animador profesional y voluntario. Análisis de contenido. Pedagogía Social. Revista Interuniversitaria, 9, pp. 161-172.

Ministerio de Educación (2011). Real Decreto 1684/2001, de 18 de noviembre, por el que se establece el Título de Técnico Superior en Animación Sociocultural y Turística y se fijan sus enseñanzas mínimas. BOE, núm. 311 de 27 de diciembre de 2011.

Ministerio de Educación y Ciencia (2006). Propuesta de directrices para la elaboración de títulos universitarios de grado y master. Madrid: Ministerio de Educación.

Pérez Serrano, G. y Sarrate Capdevila, M. L. (2013). Diversidad Cultural y ciudadanía. Hacia una Educación Superior Inclusiva. Educación $X X 1,16,1$, pp. 85-104. Doi 105944/educís.16.1.718.

Real Academia Española (2001). Diccionario de la Lengua Española (22a ed.). Madrid: Espasa Calpe.

Sáez Carreras, J. (2009). El enfoque por competencias en la formación de los educadores sociales: una mirada a su caja de herramientas. Pedagogía Social. Revista Interuniversitaria, 16, pp. 9-20

Soravilla, L., Pac, D. y Cano, J. (2011). Retos y encrucijadas para la adquisición de competencias por los profesionales de la animación sociocultural. Por un diálogo entre profesionales en el ámbito universitario. Recuperado de http://www.unizar.es/colinanimacion-IEPSA/wp-content/uploads/2011/10/4.6

\section{Notas}

1 AECID 2012. Número de referencia del Proyecto AP/036665/11

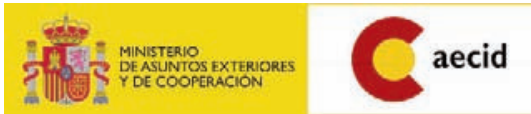

2 Se opta, en este artículo, por el uso genérico del masculino para facilitar la lectura y sin ánimo alguno de exclusión.

3 AECID 2012. Reference number AP/036665/11.

\section{Dirección de los autores}

Ma Luisa Sarrate Capdevila, José Luis García Llamas, Gloria Pérez Serrano.

Universidad Nacional de Educación a Distancia.

Departamento de Teoría de la Educación y Pedagogía Social. Facultad de Educación.

C/ Juan del Rosal, 14. 28030 Madrid.

Correo electrónico: msarrate@edu.uned.es, jlgarcia@edu.uned.es, gloriaperez@edu.uned.es

Fecha de recepción del artículo: 11.2.2013

Fecha de revisión del artículo: 25.3.2013

Fecha de aceptación final: 25.6.2013

\section{Cómo citar este artículo}

Sarrate Capdevila, M. L.; García Llamas, J. L. y Pérez Serrano, G. (2013). Exigencias profesionales del animador/a: Competencias clave. Pedagogía Social. Revista Interuniversitaria, 22, pp. 75-89. 